\title{
Time-multiplexed measurements of nonclassical light at telecom wavelengths
}

\author{
G. Harder, ${ }^{1, *}$ C. Silberhorn, ${ }^{1,2}$ J. Rehacek, ${ }^{3, \dagger}$ Z. Hradil, ${ }^{3}$ L. Motka, ${ }^{3}$ B. Stoklasa, ${ }^{3}$ and L. L. Sánchez-Soto ${ }^{2,4}$ \\ ${ }^{1}$ Department of Physics, University of Paderborn, Warburger Straße 100, 33098 Paderborn, Germany \\ ${ }^{2}$ Max-Planck-Institut für die Physik des Lichts, Günther-Scharowsky-Straße 1, Bau 24, 91058 Erlangen, Germany \\ ${ }^{3}$ Department of Optics, Palacky University, 17 listopadu 12, 77146 Olomouc, Czech Republic \\ ${ }^{4}$ Departamento de Óptica, Facultad de Física, Universidad Complutense, 28040 Madrid, Spain
}

(Received 16 June 2014; published 7 October 2014)

\begin{abstract}
We report the experimental reconstruction of the statistical properties of an ultrafast pulsed type II parametric down-conversion source in a periodically poled potassium titanyl phosphate waveguide at telecom wavelengths, with almost perfect photon-number correlations. We use a photon-number-resolving time-multiplexed detector based on a fiber-optical setup and a pair of avalanche photodiodes. By resorting to a germane data-pattern tomography, we assess the properties of the nonclassical light states with unprecedented precision.
\end{abstract}

DOI: 10.1103/PhysRevA.90.042105

PACS number(s): 03.65.Wj, 42.50.Ar, 42.50.Dv, 42.65.Wi

\section{INTRODUCTION}

Nonclassical states of light constitute an invaluable resource for deploying quantum-enhanced technologies as diverse as cryptography, computing, and metrology, to cite only some of the many relevant examples. Certifying nonclassicality requires inferring either the photon-number distribution or a quasiprobability distribution from a set of measurements. Even though the latter approach is well established [1] (it involves homodyne detection followed by an appropriate reconstruction scheme), photon counting seems a more natural choice in this discrete-variable scenario, in which photons are used as flying qubits.

Capitalizing on photon counting places stringent demands on detector performance. This is currently driving considerable improvements in single-photon detectors [2-10]; in particular, the photon-number-resolving (PNR) capability is nowadays required in most advanced protocols [11].

Several strategies have been proposed thus far for PNR detectors. Single-photon avalanche diodes (SPADs) have become the prevailing option for many applications. Si-based SPADs constitute a relatively mature technology with several efficient devices commercially available, but they are only suitable for use at visible and near-infrared wavelengths. For experiments at technologically important telecom wavelengths, the main contending technologies are InGaAs SPADs, which are plagued by high dark-count rates and long dead times, thereby making gating essential.

A proposal to employ a time-multiplexed detection (TMD) based on SPAD has been put recently forward [12-14]. These TMDs work also for pulsed light, and the photon-number distribution of a quantum state can be retrieved by inverting the measured photon statistics. Experimental applications, demonstrating a reliable loss calibration, and the TMD suitability for detecting multimode statistics and nonclassicality, have already been accomplished [15-20].

The effective implementation of these advanced schemes relies on a complete and accurate knowledge of the detector, an issue that has lately started to attract a good deal of

\footnotetext{
*georg.harder@uni-paderborn.de
}

${ }^{\dagger}$ rehacek@optics.upol.cz attention [21-27]. The idea is to employ the outcome statistics in response to a set of complete certified input states.

However, as shown in Ref. [28], if the measurement itself is of no interest, the costly detector calibration can be bypassed by using a direct fitting of data in terms of detector responses to input probes. Thus, state estimation is done without any prior knowledge of the measurement, avoiding unnecessary wasting of resources $[29,30]$.

In this paper, we demonstrate this data-pattern tomography by presenting a complete reconstruction of nonclassical states of light using an uncalibrated TMD and discussing their intriguing features.

\section{EXPERIMENTAL SETUP}

The states in our experiment are generated by type II spontaneous parametric down-conversion (SPDC) inside a periodically poled potassium titanyl phosphate (KTP) waveguide. The SPDC source produces decorrelated signal and idler states with a purity for heralded states above $80 \%$ and high coupling efficiency into single-mode fibers. The setup is the same as the one described in detail in Ref. [31] and sketched in Fig. 1, which schematizes also the TMD: two incoming pulses are split into 16 temporal bins and impinge onto SPADs. Counting the clicks allows us to estimate photon numbers and photon-number correlations between the two input ports. Since we work at telecom wavelengths, we use InGaAs SPADs (Id Quantique id201 at a repetition rate of $1 \mathrm{MHz}$ with a gate width of about $2.5 \mathrm{~ns}$ ). As briefly mentioned before, InGaAs SPADs are the simplest and most cost-efficient detectors available at telecom wavelengths. However, they have some disadvantages: the detection efficiencies are below $25 \%$ and afterpulsing is present with a few percent probability [32]. Consequently, the conventional TMD model [12], which only takes into account the probabilistic splitting and overall losses, is inadequate. A more sophisticated technique is required to recover photon statistics from the measured click frequencies; this is where data-pattern tomography comes into play.

The state is specified by the two-mode photon-number distribution $P_{m n}$, where the first (second) index refers to the signal (idler) mode. We also denote by $p_{\alpha \beta}$ the probability of simultaneous signal $(\alpha)$ and idler $(\beta)$ detection. Detections are thus described by eight-digit binary numbers, where values of 

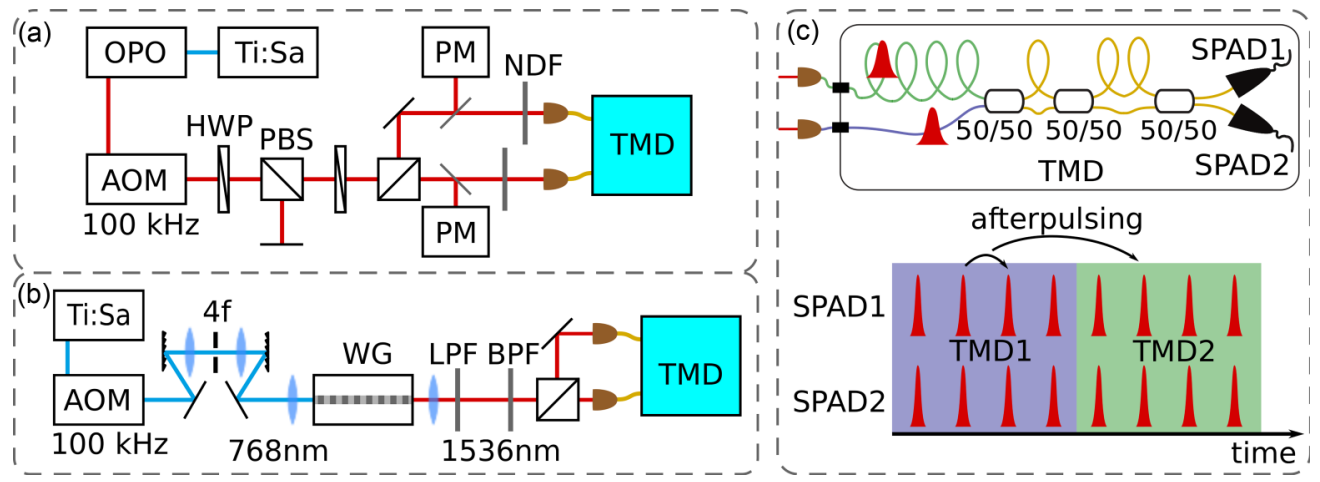

FIG. 1. (Color online) (a) Probe-state generation. Pulsed light at telecom wavelengths is generated in a Ti:sapphire pumped optical parametric oscillator (OPO). The repetition rate of the reference is lowered by an acousto-optic modulator (AOM). Motorized half-wave plates (HWP) followed by polarizing beam splitters (PBS) are used to vary the attenuation. The light power is measured by power meters (PM), further attenuated by neutral density filters (NDF), and coupled into the single-mode fibers of the time-multiplexing detector (TMD). (b) Nonclassical state generation. The pump light is spectrally tailored by a $4 f$ system, coupled into the KTP waveguide (WG) and blocked by a long pass filter. The SPDC photons pass a bandpass filter (BPF), are separated by a PBS and coupled into the TMD. (c) TMD. Two input pulses are distributed into 16 bins to obtain information about the photon number in each input as well as photon-number correlations between the beams.

0 or 1 mean click or no click in the corresponding time bin. This gives $2^{8}=256$ distinct single-mode events and $2^{16}=65536$ two-mode events to reckon with. This number is well below the limitations of our method; however, eight bins per mode seems more than enough to reveal the subtle signal features, while preventing signal-to-noise degradation. With a further splitting of the modes, the dark counts would start to play a significant role, decreasing thus the accuracy of the reconstruction. On the other hand, an effective reduction of the number of bins can always be done in postprocessing by combining the existing detection channels.

We adopt a linear model of the TMD detection, so that

$$
p_{\alpha \beta}=\sum_{m=0}^{d-1} \sum_{n=0}^{d-1} C_{\alpha \beta, m n} P_{m n},
$$

where $d$ is the cutoff dimension required to accommodate the relevant parts of the signal and the idler. The measurement matrix $C$ provides a complete description of the TMD, including losses, detector efficiencies, and afterpulsing effects.

In a real experiment, we acquire the relative frequencies $f_{\alpha \beta}$ after $N$ random samples are drawn from the multinomial distribution parametrized by $p_{\alpha \beta}$. Due to afterpulsing, it is not possible to factorize the detection matrix in signal and idler parts.

We also consider single-mode and heralded events; the former (latter) are simply marginal (conditional) probabilities of $p_{\alpha \beta}$. For these single-mode events, we look at the total number of clicks (either in the signal or the idler), without paying attention to the particular ordering of time bins. For example, for the signal-mode reconstruction, such reduction is readily done by summing data and patterns $f_{\alpha}=\sum_{\beta} f_{\alpha \beta}$ over the $8 ! /[(8-k) ! k !]$ different permutations of $\alpha$ with the same number $k$ of nonzero binary digits.

\section{FITTING DATA PATTERNS}

From the measured data $f_{\alpha \beta}$ we have to determine the state $P_{m n}$. The standard detector tomography would proceed in two steps: first, a detector estimation, where the measurement matrix $C_{\alpha \beta, m n} \geqslant 0$ is inferred from a set of calibration states. Afterwards, the state $P_{m n} \geqslant 0$ is reconstructed from the previously obtained detector matrix. However, this is not completely satisfactory: the details of the TMD are not of interest and, besides, the detector estimation is exceedingly costly, scaling as $d^{4}$, which makes the method impractical, even for moderate values of this cutoff $d$.

The alternative data-pattern approach, we adopt here, expresses $P_{m n}$ as a mixture

$$
P_{m n}=\sum_{\xi=1}^{M} x_{\xi} P_{m n}^{(\xi)}=\sum_{\xi=1}^{M} x_{\xi} P_{m}^{(\xi)} P_{n}^{(\xi)}
$$

of $M$ linearly independent (generally, nonorthogonal) twomode coherent probes $\left\{P_{m n}^{(\xi)}\right\}$, with positive and negative weights $\left\{x_{\xi}\right\}$. This can be thought of as a kind of discrete generalization of the $P$ representation and can be straightforwardly extended to any number of modes. As long as there is a match between the number of linearly independent probes and the number of free parameters, the decomposition is unique. For overcomplete set of probes, the uniqueness is not guaranteed, but all the feasible vectors $x_{\xi}$ result in the same solution. For incomplete sets, the decomposition is, in general, unfeasible, albeit partial tomography of the signal is still plausible.

We would like to stress that the actual field of view of the setup is uniquely and operationally determined by the known set of measured probes rather than some ad hoc truncation of the search space. In our experiment, the photon-number distribution of easy-to-generate coherent states, $P_{n}^{(\xi)}=|\langle n \mid \alpha(\xi)\rangle|^{2}$, where $|n\rangle$ is a Fock state, serve as the probes and hence they are termed coherent probes.

The responses $f_{\alpha \beta}^{(\xi)}$ of the TMD to these coherent probes are called patterns. Then, by linearity, the data (i.e., the TMD response $f_{\alpha \beta}$ to an unknown state $P_{m n}$ ) can be modeled as

$$
f_{\alpha \beta} \simeq \sum_{\xi=1}^{M} x_{\xi} f_{\alpha \beta}^{(\xi)}
$$


Hence, once the patterns and data are acquired (as outlined in Fig. 1), the coefficients $x_{\xi}$ can be inferred from Eq. (3) and the state reconstructed according to Eq. (2). To this end, a suitable convex measure of the distance between the leftand right-hand sides of Eq. (3) has to be minimized, subject to the physical constraints $P_{m n} \geqslant 0$ and $\sum_{m n} P_{m n}=1$ : this is a quadratic program, for which stable algorithms, polynomial in the size of the problem, are available [33].

Assuming a complete two-mode tomography $\left(M=d^{2}\right)$, the number of free parameters in the data-pattern approach scales as $d^{2}$ versus $d^{4}$ in the standard approach. Besides, the advantage of the inversion (2) over the standard postprocessing is evident. Concerning the complexity of the measurement, we underline that, in contradistinction to standard detector tomography, the number of parameters, $M$, here is independent of the cutoff dimension $d$ and, therefore, incomplete sets of probes $M \ll d^{2}$ can be measured and processed much in the same way as complete ones, significantly decreasing the measurement time. Such an incomplete tomography make sense if some prior information on signal is available. Also, if required, partial tomography can be performed by using only a small part of the probes, e.g., only a few matrix elements, or any linear function of them (such as the value of the Wigner function at the origin) for the signal reconstruction in Eq. (3). This indicates the great flexibility of this technique.

To create the probe states we use pulsed coherent light attenuated at the single-photon level. The power of the reference beam is changed by two motorized half-wave plates followed by polarizing beam splitters. We calibrate the neutral density filters separately and measure fiber-coupling losses. From these values and the measured reference power, we calculate the power inside the fibers of the TMD. Due to the high degree of attenuation (of the order of $1 \times 10^{-9}$ ), small calibration errors (of order of a few percent) cannot be avoided, although they affect the total losses but not the shape of the photon statistics.

\section{RESULTS}

We take into consideration a fixed number of patterns with amplitudes below a given threshold $\alpha_{\max } \approx 2$. This threshold is important because of the afterpulsing, which seems to be more pronounced for stronger states. The reconstruction is repeated 100 times with randomly chosen probe subsets of size $M<235$ and averaged over those repetitions. In this way, the redundancy in the data is propagated into the final estimate.

The variation within the set of reconstructions is used to estimate the associated errors, much in the spirit of nonparametric bootstrap [34]. In the experiment, $N_{\xi}=4.2 \times 10^{6}$ events were registered for each coherent probe and SPDC state. For low-intensity SPDC states, the data were averaged over five repeated data acquisitions, making a total of $N_{\text {SPDC }}=$ $21 \times 10^{6}$ events. With these numbers, the statistical noise is insignificant (except, perhaps, for heralded detections) and the reconstruction accuracy is governed by systematic errors and afterpulsing effects.

To check the performance for different parameter sets we first performed a cross-validation [35] to verify whether the estimated state is consistent with the observed data sample. To this end, we checked the quality of the reconstruction

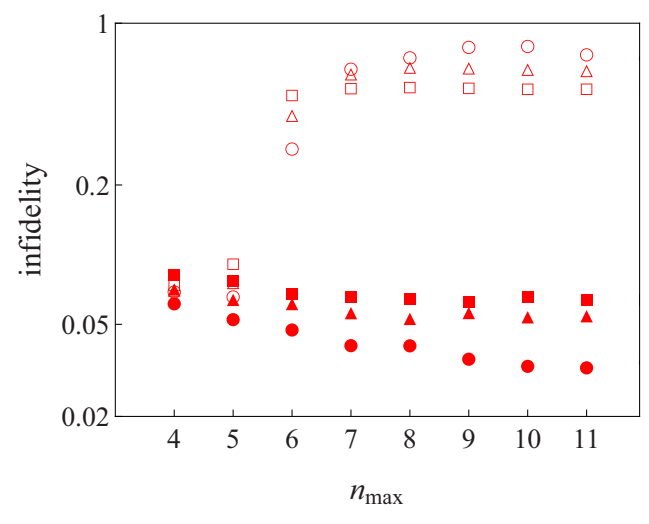

FIG. 2. (Color online) Average infidelity versus the dimension of the reconstruction space, $n_{\max }$. The number of patterns employed is indicated by symbols: squares (50 patterns), triangles ( 80 patterns), and circles (150 patterns). Open symbols are used for standard detector tomography and solid ones for data-pattern tomography.

with random sets of coherent states discarded from the probes, but with the same amplitude threshold (in our case, $\alpha<1)$. In detail: one run consisted in randomly selecting $M$ coherent states for probes and reconstructing all the remaining coherent probes (within the amplitude threshold) from the corresponding patterns. The size of the probes was varied to see the effect of the dimension. This procedure was repeated 100 times and several thousand reconstructions were done for each different size $M=50,80$, and 150 of the probe set. Finally, all the reconstructions were compared with the known true two-mode photon-number distributions.

To quantify the accuracy we employ the well-motivated infidelity

$$
1-F\left(\hat{P}_{m n}, P_{m n}\right)=1-\sum_{m n} \sqrt{\hat{P}_{m n} P_{m n}}
$$

between the estimate $\hat{P}_{m n}$ and the true state $P_{m n}$, although the same conclusions are reached with other measures. Furthermore, we apply the same procedure for the standard detector tomography, so we can perform a fair comparison.

In Fig. 2, we plot the average infidelity as a function of $n_{\max }$, which is the maximum dimension of the the matrix $P_{m n}$ (i.e., $0 \leqslant m, n \leqslant n_{\max }$ ). Except for very small reconstruction dimensions, patterns largely outperform detector tomography. Indeed, this latter technique fails for $n_{\max }>5$, whereas datapattern tomography improves remarkably as $n_{\max }$ increases. In both cases, the number of probes slightly enhances the performance. To get the data in Fig. 2, we have used linear estimation and we have ignored the positivity constraint. Once the physical constraints are incorporated, we get infidelities of the order of $1 \%$, which amounts to errors of a few percent for the reconstructed elements of $P_{m n}$.

More probes give somewhat better results, but small sets of probes can be surprisingly good. This is due to the small variation across those patterns characterized by a small number of principal components in the singular value decomposition. To compare with the theory, we assume the SPDC distribution

$$
\mathscr{P}_{m n}=\frac{\langle n\rangle^{n}}{(1+\langle n\rangle)^{1+n}} \delta_{m n},
$$



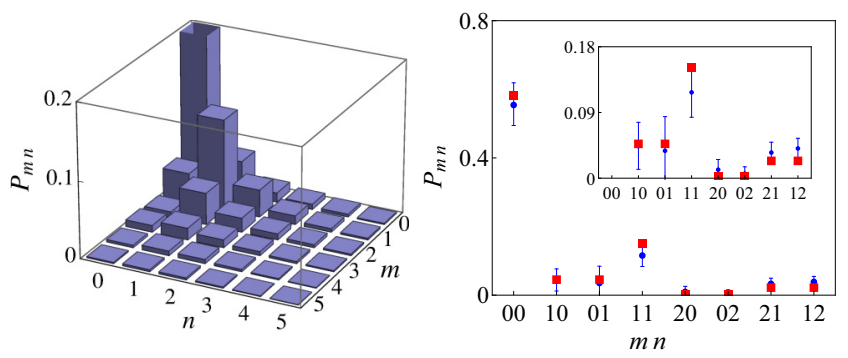

FIG. 3. (Color online) Two-mode reconstruction of the $\mathrm{SPDC}_{2}$ state. Left: Complete photon-number distribution, with $M=50$ probes. Right: Comparison with the theoretical model. Circles with error bars represent the experimental results, whereas theory is indicated by squares.

together with a finite detection efficiency $\eta$ which is taken into account by a Bernoulli distribution:

$$
P_{m n}=\sum_{k=m}^{\infty} \sum_{\ell=n}^{\infty}\left(\begin{array}{l}
k \\
m
\end{array}\right)\left(\begin{array}{l}
\ell \\
n
\end{array}\right) \eta^{n+m}(1-\eta)^{k+\ell-n-m} \mathscr{P}_{k \ell} .
$$

From the zero-detection probabilities of coherent probes with known amplitudes, the quantum efficiency of detectors was estimated to be $0.22 \pm 0.01$ and the coupling efficiency $75 \%$. This, in turn, enables us to calculate the mean photon numbers of the SPDC states: we generated three different ones, denoted $\mathrm{SPDC}_{1}, \mathrm{SPDC}_{2}$, and $\mathrm{SPDC}_{3}$, with $\left\langle n_{1}\right\rangle=0.11,\left\langle n_{2}\right\rangle=0.76$, and $\left\langle n_{3}\right\rangle=1.34$, respectively. These numbers were used to predict the two-mode statistics through Eqs. (5) and (6). In Fig. 3 we plot typical results of two-mode TMD measurements for $\mathrm{SPDC}_{2}$. Strong signal-idler correlations are observed and the agreement with the theory is pretty good. Similar results are found for other intensities. In Fig. 4 we show the reconstructions of the signal states for two different pump intensities. Best fits to Bose-Einstein distributions are almost indistinguishable from the experimental results.

Heralded states are created by having the idler state conditioned on single or double detection in the signal. By double detection we mean here a click at detector $A$ accompanied by a simultaneous click at detector $B$. Double detections at any single detector are discarded to avoid doubles caused by afterpulsing. Heralded single- and especially two-photon states are difficult to reconstruct, since we are picking out

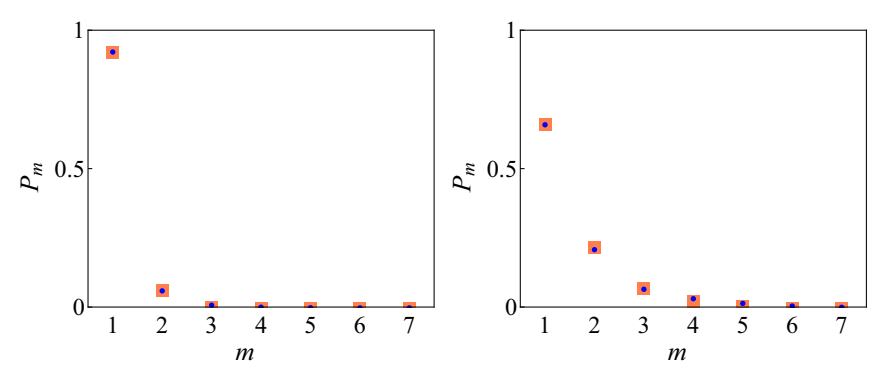

FIG. 4. (Color online) Single-mode signal reconstructions (dots) of SPDC $_{1}$ (left) and $\mathrm{SPDC}_{2}$ (right), both with $M=30$ probes. Best fits to Bose-Einstein distributions (squares) are also shown. The reconstruction uncertainties are almost negligible and cannot be appreciated.
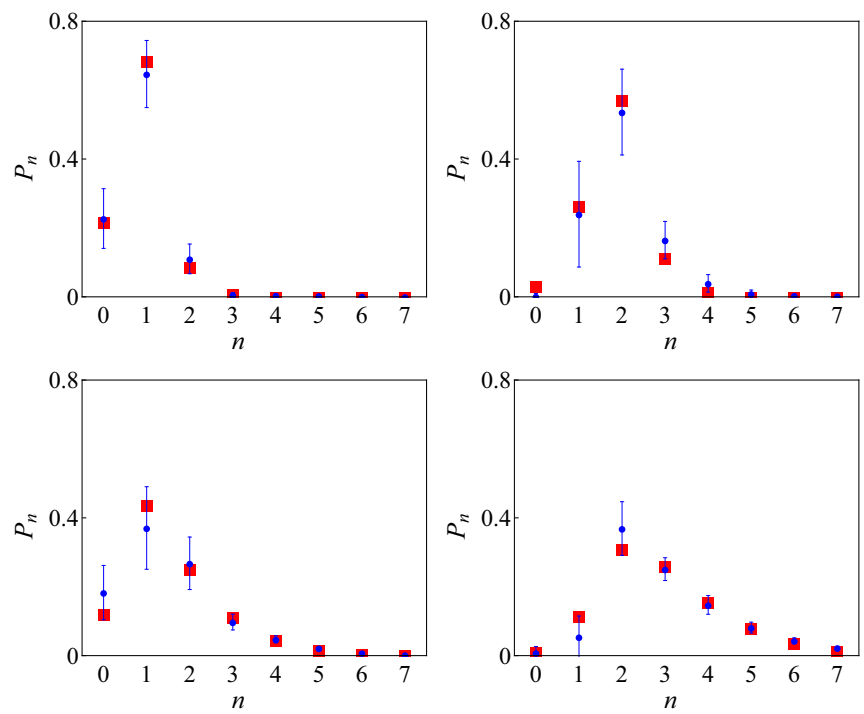

FIG. 5. (Color online) Reconstructed single-photon (left) and two-photon (right) heralded idler states generated from $\mathrm{SPDC}_{1}$ (top) and $\mathrm{SPDC}_{2}$ (bottom), with $M=80$ probes. Squares denote again the corresponding theoretical predictions.

quite a small subset of all the detection events. Besides, afterpulsing creates artificial signal-idler correlations, whose strength depends on the distance of the signal detection from the first idler time bin. This leads to larger reconstruction errors.

Reconstructed single- and two-photon heralded idler states from two different SPDC states are shown in Fig. 5. To get theoretical predictions, we again assume an inefficient coupling (0.75) of the SPDC state and calculate the postmeasurement idler state $P_{i}$ from the premeasurement $P$ as

$$
P_{i}=\frac{\operatorname{Tr}_{s}\left(E P E^{\dagger}\right)}{\operatorname{Tr}_{s, i}\left(E P E^{\dagger}\right)},
$$

where $\hat{E}^{\dagger} \hat{E}$ is the positive operator-valued measure (POVM) element describing the single or double detection in the signal mode and $\operatorname{Tr}_{s, i}$ indicates trace over the signal or idler. All states and POVM elements are diagonal here.

Best estimates of the Wigner function at the origin for the single-photon heralded states are $W(0)=-0.72 \pm 0.06$ $\left(\mathrm{SPDC}_{1}\right)$ and $W(0)=-0.30 \pm 0.09\left(\mathrm{SPDC}_{2}\right)$. This agrees with the calculated values $W(0)=-0.77 \quad\left(\mathrm{SPDC}_{1}\right)$ and $W(0)=-0.29\left(\mathrm{SPDC}_{2}\right)$, respectively, and confirms the nonclassicality of these states. With more intense SPDC inputs, single detection in the signal tends to leave a mixture of Fock states in the idler. This explains why the nonclassicality of heralded states decreases with increasing pump intensity.

Finally, we also simulated heralded states as postmeasurement states based on the results of full two-mode tomography. To this end, we performed 100 two-mode reconstructions for each measured SPDC state. The idler postmeasurement state is calculated based on a presumed single or double signal detection. The statistics of the resulting ensemble of heralded states is shown in Fig. 6, along with the theoretical predictions.

These predictions based on the full two-mode reconstructions are less accurate than the single-mode heralded ones. 

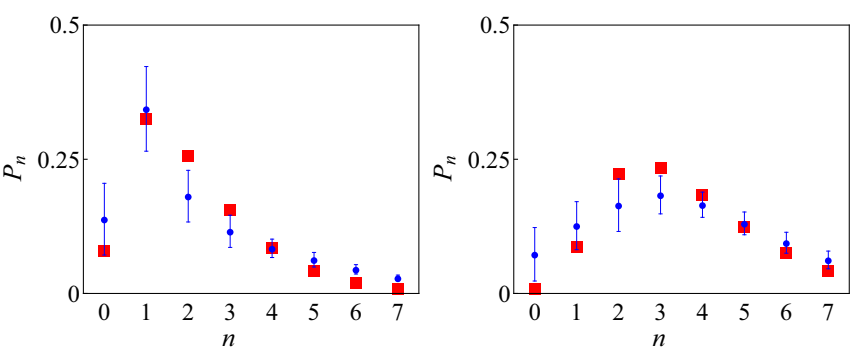

FIG. 6. (Color online) Heralded single-photon (left) and doublephoton (right) idler states as predicted from the reconstructed twomode photon-number distributions of $\mathrm{SPDC}_{3}$, with $M=80$ probes.

The latter is more direct. In heralded detections, what helps is that the dimension of the search space is reduced and the dominating vacuum or even single-photon terms are eliminated, which improves the accuracy. In addition, in the data-pattern approach we use heralded coherent probes; i.e., we do the same data selection as for the SPDC data. In this way, one somehow eliminates the artificial correlations created by the afterpulsing. Nevertheless, it is nice to see that the agreement between single- and two-mode measurements is actually pretty good. The two-mode predictions improve with increasing intensity, as one could expect. More intense SPDC states have larger higher-order $P_{m n}$ components, which are easier to extract.

\section{CONCLUDING REMARKS}

The experimental probing of a TMD device with cheap coherent inputs and its subsequent use in the reconstruction of nonclassical twin beams from SPDC presented here not only demonstrates a successful implementation of this type of data-pattern tomography, but also goes far beyond the standard quantum detector tomography. The approach is easily adapted to a variety of measurement devices: the recent implementation in Ref. [30] and the experimental implementation presented here show its viability for complex detectors.

\section{ACKNOWLEDGMENTS}

J.R., Z.H., L.M., and B.S. are thankful for the financial assistance of the European Social Fund and the State Budget of the Czech Republic POSTUP II (Grant No. CZ.1.07/2.3.00/30.0041), Technology Agency of the Czech Republic (Grant No. TE01020229), and the IGA Project of the Palacký University (Grant No. PRF 2013 019). L.L.S.S acknowledges the support from the Spanish MINECO (Grant No. FIS2011-26786).
[1] A. I. Lvovsky and M. G. Raymer, Rev. Mod. Phys. 81, 299 (2009).

[2] R. H. Hadfield, Nat. Photonics 3, 696 (2009).

[3] G. S. Buller and R. J. Collins, Meas. Sci. Technol. 21, 012002 (2010).

[4] M. D. Eisaman, J. Fan, A. Migdall, and S. V. Polyakov, Rev. Sci. Instrum. 82, 071101 (2011).

[5] J. P. Sprengers, A. Gaggero, D. Sahin, S. Jahanmirinejad, G. Frucci, F. Mattioli, R. Leoni, J. Beetz, M. Lermer, M. Kamp, S. Höfling, R. Sanjines, and A. Fiore, Appl. Phys. Lett. 99, 181110 (2011).

[6] C. M. Natarajan, M. G. Tanner, and R. H. Hadfield, Supercond. Sci. Technol. 25, 063001 (2012).

[7] W. H. P. Pernice, C. Schuck, O. Minaeva, M. Li, G. N. Goltsman, A. V. Sergienko, and H. X. Tang, Nat. Commun. 3, 1325 (2012).

[8] B. Calkins, P. L. Mennea, A. E. Lita, B. J. Metcalf, W. S. Kolthammer, A. Lamas-Linares, J. B. Spring, P. C. Humphreys, R. P. Mirin, J. C. Gates, P. G. R. Smith, I. A. Walmsley, T. Gerrits, and S. W. Nam, Opt. Express 21, 22657 (2013).

[9] Single-Photon Generation and Detection, edited by A. Migdall, S. V. Polyakov, J. Fan, and J. C. Bienfang (Academic Press, Oxford, 2013).

[10] C. M. Natarajan, L. Zhang, H. Coldenstrodt-Ronge, G. Donati, S. N. Dorenbos, V. Zwiller, I. A. Walmsley, and R. H. Hadfield, Opt. Express 21, 893 (2013).

[11] J. L. O'Brien, A. Furusawa, and J. Vuckovic, Nat. Photonics 3, 687 (2009).

[12] D. Achilles, C. Silberhorn, C. Śliwa, K. Banaszek, and I. A. Walmsley, Opt. Lett. 28, 2387 (2003).

[13] J. Řeháček, Z. Hradil, O. Haderka, J. Peřina, and M. Hamar, Phys. Rev. A 67, 061801 (2003).
[14] M. J. Fitch, B. C. Jacobs, T. B. Pittman, and J. D. Franson, Phys. Rev. A 68, 043814 (2003).

[15] D. Achilles, C. Silberhorn, and I. A. Walmsley, Phys. Rev. Lett. 97, 043602 (2006).

[16] M. Avenhaus, H. B. Coldenstrodt-Ronge, K. Laiho, W. Mauerer, I. A. Walmsley, and C. Silberhorn, Phys. Rev. Lett. 101, 053601 (2008).

[17] A. P. Worsley, H. B. Coldenstrodt-Ronge, J. S. Lundeen, P. J. Mosley, B. J. Smith, G. Puentes, N. Thomas-Peter, and I. A. Walmsley, Opt. Express 17, 4397 (2009).

[18] M. Avenhaus, K. Laiho, M. V. Chekhova, and C. Silberhorn, Phys. Rev. Lett. 104, 063602 (2010).

[19] K. Laiho, K. N. Cassemiro, D. Gross, and C. Silberhorn, Phys. Rev. Lett. 105, 253603 (2010).

[20] T. J. Bartley, G. Donati, X.-M. Jin, A. Datta, M. Barbieri, and I. A. Walmsley, Phys. Rev. Lett. 110, 173602 (2013).

[21] A. Luis and L. L. Sánchez-Soto, Phys. Rev. Lett. 83, 3573 (1999).

[22] J. Fiurášek, Phys. Rev. A 64, 024102 (2001).

[23] G. M. D'Ariano, L. Maccone, and P. Lo Presti, Phys. Rev. Lett. 93, 250407 (2004).

[24] J. S. Lundeen, A. Feito, H. Coldenstrodt-Ronge, K. L. Pregnell, C. Silberhorn, T. C. Ralph, J. Eisert, M. B. Plenio, and I. A. Walmsley, Nat. Phys. 5, 27 (2009).

[25] T. Amri, J. Laurat, and C. Fabre, Phys. Rev. Lett. 106, 020502 (2011).

[26] L. Zhang, A. Datta, H. B. Coldenstrodt-Ronge, X.-M. Jin, J. Eisert, M. B. Plenio, and I. A. Walmsley, New J. Phys. 14, 115005 (2012).

[27] G. Brida, L. Ciavarella, I. P. Degiovanni, M. Genovese, L. Lolli, M. G. Mingolla, F. Piacentini, M. Rajteri, E. Taralli, and M. G. A. Paris, New J. Phys. 14, 085001 (2012). 
[28] J. Řeháček, D. Mogilevtsev, and Z. Hradil, Phys. Rev. Lett. 105, 010402 (2010).

[29] D. Mogilevtsev, A. Ignatenko, A. Maloshtan, B. Stoklasa, J. Rehacek, and Z. Hradil, New J. Phys. 15, 025038 (2013).

[30] M. Cooper, M. Karpiński, and B. J. Smith, Nat. Commun. 5, 4332 (2014).

[31] G. Harder, V. Ansari, B. Brecht, T. Dirmeier, C. Marquardt, and C. Silberhorn, Opt. Express 21, 13975 (2013).
[32] Y. Kang, H. Lu, Y.-H. Lo, D. Bethune, and W. Risk, Appl. Phys. Lett. 83, 2955 (2003).

[33] L. Motka, B. Stoklasa, J. Rehacek, Z. Hradil, V. Karasek, D. Mogilevtsev, G. Harder, C. Silberhorn, and L. L. SánchezSoto, Phys. Rev. A 89, 054102 (2014).

[34] A. C. Davison and D. V. Hinkley, Bootstrap Methods and Their Application (Cambridge University Press, Cambridge, UK, 1997).

[35] D. Mogilevtsev, Z. Hradil, J. Rehacek, and V. S. Shchesnovich, Phys. Rev. Lett. 111, 120403 (2013). 